\title{
Antisense PNA effects in Escherichia coli are limited by the outer-membrane LPS layer
}

\author{
Liam Good, ${ }^{1}$ Rickard Sandberg, ${ }^{1}$ Ola Larsson, ${ }^{1}$ Peter E. Nielsen ${ }^{2}$ \\ and Claes Wahlestedt ${ }^{1}$
}

Author for correspondence: Liam Good. Tel: +46 8728 6697. Fax: +46 8323950.

e-mail: liam.good@cgr.ki.se

\footnotetext{
1 Center for Genomics Research, Karolinska Institute, Berzelius väg 37, 171 77, Stockholm, Sweden

2 Center for Biomolecular Recognition, Panum Institute, Blegdamsvej 3c, DK 2200 Copenhagen N., and Pantheco A/S, Fruebjergvej 3, DK 2100 Copenhagen Ø., Denmark
}

\begin{abstract}
Antisense peptide nucleic acids (PNAs) can inhibit Escherichia coli gene expression and cell growth through sequence-specific RNA binding, and this opens possibilities for novel anti-infective agents and tools for microbial functional genomics. However, the cellular effects of PNAs are limited relative to effects in cell extracts, presumably because of cell barrier components such as the outer-membrane lipopolysaccharide (LPS) layer or drug efflux pumps, both of which function to exclude antibiotics and other foreign molecules. To evaluate the importance of such cellular factors on PNA effects, the authors developed a positive assay for antisense inhibition by targeting the lac operon repressor and compared PNA susceptibilities in mutant and wild-type $E$. coli by assessing lacZ induction. Strains with defective LPS (AS19 and D22) were more permeable to the antibiotic nitrocefin and more susceptible to PNA than the wild-type. Also, PNA potency was improved in wild-type cells grown in the presence of certain cell-wall-permeabilizing agents. In contrast, the activities of the Acr and Emr drug efflux pumps were not found to affect PNA susceptibility. The results show that the LPS layer is a major barrier against cell entry, but PNAs that can enter $E$. coli are likely to remain active inside cells.
\end{abstract}

Keywords: peptide nucleic acid, bacteria, lipopolysaccharide

\section{INTRODUCTION}

Bacteria possess endogenous antisense gene control mechanisms (Simons \& Kleckner, 1988), and recent experiments show that antisense gene inhibition in bacteria is possible using synthetic antisense agents. Peptide nucleic acids (PNAs) targeted to RNA components of the ribosome can inhibit translation and cell growth, and PNAs targeted to mRNA can limit gene expression with gene and sequence specificity (Good \& Nielsen, 1998a, b). Therefore, PNAs can provide tools for functional analyses of bacterial genes and there are attractive possibilities for novel sequence-designed antiinfective agents (Good \& Nielsen, 1998a, b; Ecker \& Freier, 1998). To improve the approach, the challenge is to learn more about cellular limits to PNA activity and design molecules to more efficiently enter cells and access RNA targets.

PNA is a DNA mimic with nucleobases attached to a

Abbreviations: CCCP, carbonyl cyanide $m$-chlorophenylhydrazone; PNA, peptide nucleic acid. pseudopeptide backbone (Nielsen et al., 1991) (Fig. 1a). PNA hybridizes to complementary DNA, RNA or PNA oligomer sequences through Watson and Crick base pairing and helix formation (Egholm et al., 1993; Wittung et al., 1994; Jensen et al., 1997). The peptide backbone provides improved hybridization affinity and specificity properties (Egholm et al., 1993; Jensen et al., 1997), resistance to enzymic degradation (Demidov et al., 1994) and access to a variety of chemical modifications (Nielsen \& Haaima, 1997; Püschl et al., 1998). For antisense applications, target-bound PNA can hinder the activities of DNA and RNA polymerases, reverse transcriptase, telomerase and the ribosome (Hanvey et al., 1992; Knudsen \& Nielsen, 1996; Good \& Nielsen, 1998a). Also, in vitro assays for telomerase activity and mitochondrial genome replication show that PNAs are often effective in applications where other (modified) oligonucleotides perform poorly (Norton et al., 1996; Taylor et al., 1997).

Limited cellular uptake is a general difficulty with antisense agents. Furthermore, restricted uptake is expected with Escherichia coli because of stringent 
cellular barriers against foreign molecules (Nikaido, 1994). The outer barrier of Gram-negative bacteria consists of a peptidoglycan layer, a lipid bilayer and an external lipopolysaccharide (LPS) coating. Furthermore, foreign compounds that do enter cells can be exported by various translocases, which are believed to "pump out' a wide range of foreign compounds including certain peptides (Nikaido, 1994; Lewis, 1994). Therefore, typical bacterial cells are well equipped to resist foreign molecules, such as PNA. Indeed, results from cell extract and cell culture experiments suggest that only a small proportion of PNAs can enter and act within cells (Good \& Nielsen, 1998a, b).

To study factors that limit PNA antisense effects apart from nonspecific inhibition, we designed a PNA to target the lac repressor mRNA. Limited repressor expression was seen as induced $\beta$-galactosidase (LacZ) activity in E. coli cells and taken as an indicator of antisense effect. The anti-lac repressor PNA was used together with mutant $E$. coli strains and chemicals known to disrupt cell wall components. The results show that the outer-membrane LPS layer is a major limit to PNA cell entry and antisense effects in E. coli.

\section{METHODS}

Bacteria and plasmids. The E. coli strains used in this study are listed in Tables 1 and 3. Plasmid pBX1, which carries a wild-type copy of the acr operon, was obtained from Kevin Bertrand (Washington State University), and plasmid pEMR2.1, which carries a wild-type copy of the emrB drug translocase, was obtained from Kim Lewis (Tufts University).

PNAs and other chemicals. The PNAs used in this study were synthesized as described by Christensen et al. (1995), and their structures are shown in Fig. 1. The cell-wall-active chemicals were from Sigma and the nitrocefin was from Oxoid.

Bacterial growth conditions. Growth on solid media was initiated with an inoculum of $10^{4}$ cells $\mathrm{ml}^{-1}$ in an overlay of agar media, and growth in liquid media was initiated with an inoculum of $10^{4}$ cells $\mathrm{ml}^{-1}$ in $200 \mu \mathrm{l}$ cultures in siliconized polypropylene microdilution tubes. The cultures were grown in $10 \%$ Luria-Bertani medium at $37^{\circ} \mathrm{C}$ for $18 \mathrm{~h}$. The chemicals listed in Table 2 were included in liquid cultures over the nanomolar concentration range. PNAs and the chemicals listed in Table 2 were added to cultures prior to overnight growth.

$\boldsymbol{\beta}$-Galactosidase activity assays. To indicate LacZ activity in solid cultures, the chromogenic substrate X-Gal was included in the agar plates at $250 \mu \mathrm{g} \mathrm{ml}^{-1}$ and $4 \mu \mathrm{l}$ PNA aliquots of PNA were applied by pipette directly onto the agar surface before growth. $\beta$-Galactosidase activity in liquid cultures was measured by using the chromogenic LacZ substrate ONPG, essentially as described by Miller (1972).

Nitrocefin outer cell barrier permeability assay. Outermembrane permeability was determined essentially as described by Angus (1982). E. coli cells transformed with pBR322 were cultured to mid-exponential phase, recovered by centrifugation at $3000 \mathrm{~g}$ and rinsed with $5 \mathrm{mM}$ HEPES (7•4). Reactions were in 96-well microtitre plates containing $0 \cdot 1$ $\mathrm{OD}_{550}$ units of cells in $5 \mathrm{mM}$ HEPES (7.4), $5 \mathrm{mM}$ carbonyl

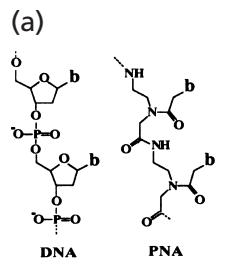

(b)

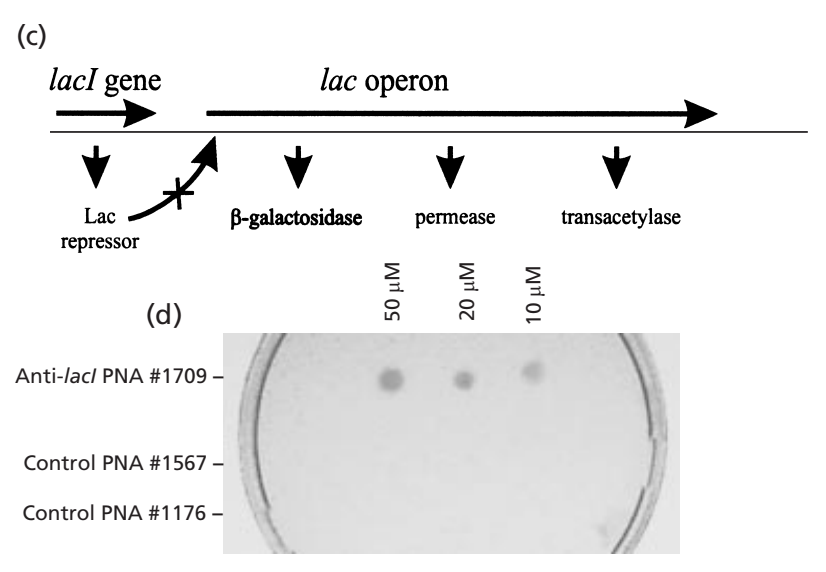

E. coli lacI (repressor) target

5' . TCAGGGTGGTAATGTG. 3' lacI mRNA GTCCCACCACTTACA- (N) anti-lacI PNA\#1709 GAATCGACATAGGCC-(N) control PNA\#1567 AGGTGTCAGCGAACG- (N) control PNA\#1176

Fig. 1. PNA-mediated induction of lacZ. (a) Structure of PNA; ' $b$ ' represents a purine or pyrimidine base. (b) Sequence of the lac repressor mRNA start codon region (start codon GUG underlined) and the antisense and control PNAs. (c) Structure of the lac operon and the PNA-mediated induction strategy. (d) PNA-mediated up-regulation of the lac operon $E$. coli strain AS19 grown on agar medium. Antisense and control PNAs were applied by direct pipetting onto overlay cultures before overnight growth. The chromogenic LacZ substrate X-Gal was included at $250 \mu \mathrm{g} \mathrm{ml}^{-1}$ and PNA solutions were applied by pipette directly onto the agar surface before growth by using $4 \mu \mathrm{l}$ applications. $\beta$-Galactosidase induction by the anti-lacl PNA is visible as blue zones against the lawn of cells with repressed lacZ expression (top row).

cyanide $m$-chlorophenylhydrazone (CCCP), and $20 \mu \mathrm{g}$ nitrocefin $\mathrm{ml}^{-1}$. Nitrocefin cleavage was monitored by measuring $A_{500}$.

\section{RESULTS AND DISCUSSION}

\section{PNA-mediated up-regulation of the lac operon}

To establish a positive assay for PNA antisense effects, we designed a new PNA (\#1709) to target the start codon region of the E. coli lac repressor gene (lacI). The lacI gene normally represses the lac operon and limits expression of the lacZ gene (Miller, 1972). To test the potential of the anti-lacl antisense strategy, PNA \#1709 was applied to a lawn of E. coli AS19 cells grown on an LB agar plate containing the LacZ substrate X-Gal, but not the lac operon inducer IPTG (Fig. 1). Increased lacZ expression was apparent as blue zones at sites of antisense PNA application (Fig. 1). Control PNAs did not up-regulate lacZ. Therefore, induction of lacZ by PNA \#1709 provides a positive assay for PNA antisense effects. The assay can be used to assess factors that limit 
Table 1. PNA-mediated lacZ induction and outer-membrane permeability of various E. coli strains

The table includes data for wild-type E. coli and strains with LPS defects. Bacteria were cultured with $2 \mu \mathrm{M}$ PNA \#1709. Values for lacZ induction show the level of enzyme activity in cultures treated with PNA over the level for untreated cultures. Cell permeation by nitrocefin is indicated as the time in minutes to observe a $50 \%$ change from the initial to final $A_{500}\left(t_{1 / 2}\right)$. The values are means \pm SD for three replicates. The F42 episome was introduced into strain D22 by mating to confer an active lac operon (Miller, 1972). Outer-membrane permeability to nitrocefin (Angus et al., 1982) was determined using cells carrying the $\beta$-lactamase gene within pBR322. CGSC indicates the Coli Genetic Stock Center, Yale University.

\begin{tabular}{|lcccl|}
\hline Strain & $\begin{array}{c}\text { Relevant } \\
\text { phenotype or } \\
\text { genotype }\end{array}$ & $\begin{array}{c}\text { lac } \boldsymbol{Z} \text { induction } \\
\text { (PNA treated/ } \\
\text { untreated })\end{array}$ & $\begin{array}{c}\text { Cell permeation } \\
\text { by nitrocefin } \\
\left(\boldsymbol{t}_{\mathbf{1} / 2}, \text { min }\right)\end{array}$ & \multicolumn{1}{c|}{ Source } \\
\hline K-12 & & $1 \cdot 3 \pm 0 \cdot 3$ & $30 \pm 6$ & CGSC 5743 \\
B & & $0 \cdot 9 \pm 0 \cdot 2$ & $29 \pm 5$ & CGSC 6739 \\
ML35 & & $1 \cdot 2 \pm 0 \cdot 6$ & $34 \pm 4$ & R. Lehrer, UCLA \\
AS19 & LPS defect & $9 \cdot 0 \pm 0 \cdot 9$ & $4 \cdot 0 \pm 1$ & S. Pedersen, University of \\
& & $3 \cdot 0 \pm 0 \cdot 8$ & $10 \pm 2$ & Copenhagen \\
D22 & LPS defect & & & \\
& $($ env $A)$ & & & \\
\hline
\end{tabular}

or improve the antisense approach, apart from nonspecific inhibitory effects.

\section{LPS layer and limited antisense effects}

The outer cell membrane of Gram-negative bacteria is coated with an LPS layer that stringently limits the influx of foreign molecules (Nikaido, 1994; Hancock, 1997), and this barrier also may restrict PNA. In our previous experiments, efficient inhibition in vitro was observed with PNA concentrations in the nanomolar range, whereas growth inhibition in cells required micromolar concentrations, dilute growth medium and the cell-wall-deficient strain AS19 (Good \& Nielsen, $1998 \mathrm{a}, \mathrm{b})$. These results suggest that cell uptake, rather than limited intracellular activities, is the major limit against the use of antisense PNAs in bacteria. Therefore, we aimed to determine whether PNA susceptibility correlates with outer-barrier permeability and whether PNA antisense effects can be improved by compromising the integrity of the LPS. It was hoped that the results would clarify the need for improved uptake properties in the next generation of antisense PNAs.

Wild-type and permeable mutant E. coli strains were tested for susceptibility to antisense PNA \#1709. Among the strains listed in Table 1, AS19 and to a lesser extent D22 showed increased induction of lacZ by PNA \#1709; both of these susceptible strains are LPS defective. Strain AS19 has a severely depleted LPS layer (Zorzopulos et al., 1989) and is more susceptible to antibiotics (Sekiguchi \& Iida, 1967). Also, this strain is unusually susceptible to PNAs targeted to the rRNA and reporter gene mRNAs (Good \& Nielsen, 1998b). Strain D22 is defective in the lipid A component of its LPS and

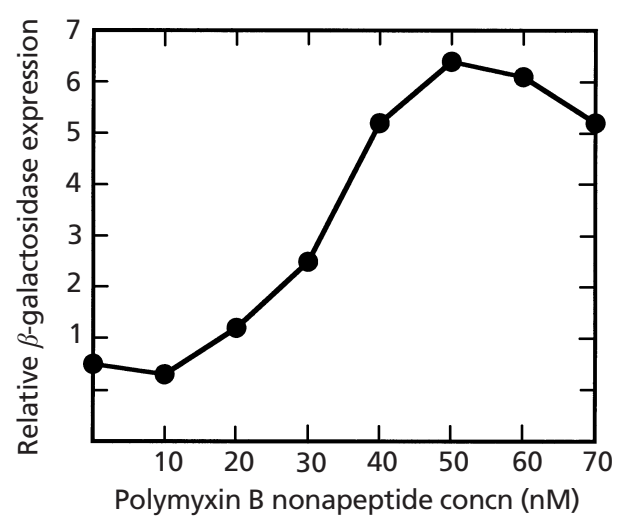

Fig. 2. Antisense PNA induction of the lac operon and effect of polymyxin B nonapeptide. The cell wall permeability increasing agent polymyxin $B$ nonapeptide was included in cultures of wild-type E. coli $\mathrm{K}-12$ containing $2 \mu \mathrm{M}$ anti-lacl PNA \#1709. Induction of the lac operon was measured by assaying LacZ activity after growth in the presence and absence of PNA $(2 \mu \mathrm{M})$ and a range of polymyxin $B$ nonapeptide concentrations (see also Table 1).

more susceptible to antibiotics (Normark et al., 1969). Therefore, LPS defects in E. coli appear to confer unusual PNA susceptibility.

To establish a more direct link between LPS integrity and PNA susceptibility for strains AS19 and D22, we tested outer-barrier permeability to nitrocefin, a chromogenic cephaloporin antibiotic. Nitrocefin is normally excluded from cells, but nitrocefin molecules that do permeate the outer membrane are cleaved by periplasmic $\beta$-lactamase and undergo a change in absorbance maxi- 
Table 2. PNA-mediated lacZ induction in the presence of cell-wall-active agents

The relative level of $l a c Z$ induction in $E$. coli $\mathrm{K}-12$ cells grown in the presence of subinhibitory concentrations of cell-active agents is shown. Bacteria were cultured with $2 \mu \mathrm{M}$ PNA \#1709. Relative values indicate the increase in LacZ activity for cultures containing added chemical agents relative to control cultures lacking added chemical agents. The values are means \pm SD for three replicates. The chemical agents were tested over a range of concentrations; the most effective are shown in parentheses. As an example, lac $Z$ induction in the presence of polymyxin $\mathrm{B}$ nonapeptide is shown in Fig. 2.

\begin{tabular}{|lll|}
\hline Chemical & $\begin{array}{c}\text { Relative } \text { lac } \boldsymbol{Z} \\
\text { induction }\end{array}$ & $\begin{array}{c}\text { Reference for } \\
\text { permeabilizing activity }\end{array}$ \\
\hline Ampicillin* & $1 \pm 0 \cdot 1$ & Sieradzki \& Tomasz (1997) \\
Cefotaxime* & $1 \pm 0 \cdot 1$ & Sieradzki \& Tomasz (1997) \\
EDTA & $1 \pm 0 \cdot 1$ & Gerberick \& Castric (1980) \\
Polymyxin B nonapeptide & $6 \pm 1 \cdot 0(50 \mathrm{nM})$ & Chunhong et al. $(1998)$ \\
Polymyxin B sulfate & $2 \pm 0 \cdot 9(20 \mathrm{nM})$ & Chunhong et al. $(1998)$ \\
Polethyleneimine & $1 \pm 0 \cdot 1$ & Helander et al. $(1997)$ \\
Prochlorperazine & $2 \pm 0 \cdot 4(50 \mathrm{nM})$ & Motohashi et al. $(1997)$ \\
Promethazine & $1 \pm 0 \cdot 1$ & Motohashi et al. $(1997)$ \\
Sodium hexametaphosphate & $1 \pm 0 \cdot 3$ & Vaara \& Jaakkola (1989) \\
\hline
\end{tabular}

*Asterisks indicate chemicals that compromise formation of the peptidoglycan layer; all other agents listed permeabilize the outer cell wall.

mum from $385 \mathrm{~nm}$ to 490 (Angus et al., 1982). E. coli strains were transformed with pBR322, selected for ampicillin resistance and subjected to the nitrocefin permeability assay (Table 1). To block respiration and eliminate the potential for active efflux of nitrocefin, CCCP was included at $5 \mu \mathrm{M}$. The results showed high outer cell barrier permeability for strain AS19 and to a lesser extent for strain D22 (Table 1, Fig. 2). These strains also showed high PNA susceptibilities (Table 1). The link between PNA susceptibility and LPS permeability suggests that the integrity of the LPS layer is a major determinant of PNA entry into E. coli.

To further investigate the importance of the LPS layer in the susceptibility of E. coli to PNA, we asked whether PNA antisense effects in wild-type E. coli strain K-12 can be improved by using chemicals that compromise LPS integrity. The anti-lac repressor PNA \#1709 was used in combination with a range of compounds that permeabilize E. coli and can increase the uptake or potency of standard antibiotics (Table 2). Several of these agents enhanced PNA potency; the most effective was polymyxin B nonapeptide, which enhanced lac $Z$ induction by approximately sixfold when present at low nanomolar concentrations (Fig. 2). An important factor in the effectiveness of polymyxin B nonapeptide in this application may be its ability to permeabilize the outer membrane with relatively low toxicity to E. coli (Chunhong et al., 1998). Antibiotics that block peptidoglycan formation did not enhance PNA effects (Table 2). Synergism between polymyxin B nonapeptide and antisense PNAs is unlikely to be useful in practical applications, but it is important as further evidence that the LPS is a major barrier to PNA uptake.

\section{Multidrug resistance translocase activity and PNA susceptibility}

Intracellular accumulation of PNAs and antisense effects also could be limited by multidrug resistance translocases, which can clear a diverse range of foreign agents from cells, including peptide antibiotics (Shafer et al., 1998). Several drug translocases are associated with multidrug-resistance phenotypes in bacteria, and these 'efflux pumps' display surprising substrate flexibility. Therefore, we investigated whether the best-known $E$. coli drug pumps could limit PNA-mediated effects. The level of lacZ induction by PNA \#1709 was determined in E. coli strains with altered drug efflux activities. Strains with defective copies of the $a c r A B$ or emrA transporter genes did not differ from the wild-type in susceptibility to PNAs (Table 3). Also, plasmids bearing cloned copies of the $\operatorname{acr} A, B$ and $e m r B$ transporter genes were introduced by transformation to complement the defective endogenous genes in strains N43 and SJ261, and active copies of these genes did not alter PNA susceptibilies (Table 3).

The acr and emr drug translocase genes are among the best-characterized E. coli drug pumps, but other translocases also could affect PNA susceptibility. A range of translocases are implicated in drug resistance and there may be as many as $18 \mathrm{ABC}$-type transporter homologues within the E. coli genome (Blattner et al., 1997). Accordingly, drug translocase involvement in PNA susceptibility was tested in a more general way using the inhibitors verapamil and reserpine, which can reduce drug efflux in E. coli and other types of cells (Beja \& Bibi, 1996; Ng et al., 1994). Neither of these compounds altered the PNA susceptibility of E. coli K-12. Therefore, 
Table 3. PNA-mediated lacZ induction in various $E$. coli strains with altered translocase activities

The table includes data for wild-type E. coli and strains with defects in translocase genes associated with drug efflux. The level of PNA-mediated lacZ induction shows the level of enzyme activity in cells treated with PNA relative to untreated cells. Bacteria were cultured with $2 \mu$ M PNA \#1709. The values are means \pm SD for three replicates.

\begin{tabular}{|c|c|c|c|}
\hline Strain & Relevant phenotype or genotype & $\begin{array}{l}\text { lac } Z \text { induction } \\
\text { (PNA treated/ } \\
\text { untreated) }\end{array}$ & Source \\
\hline K-12 & & $1 \cdot 3 \pm 0 \cdot 3$ & CGSC 5743 \\
\hline N43 & Translocase defect $(\operatorname{acr} A)$ & $1 \cdot 4 \pm 0 \cdot 2$ & CGAS 5583 \\
\hline N2616 & Translocase defect $(a c r A)$ & $0 \cdot 8 \pm 0 \cdot 2$ & CGSC 6907 \\
\hline SJ261 & Translocase defect $(e m r B)$ & $1 \cdot 1 \pm 0 \cdot 3$ & $\begin{array}{l}\text { Kim Lewis, Tufts } \\
\text { Univ., Medford, } \\
\text { MA }\end{array}$ \\
\hline PM61R & Translocase defect $(a c r E)$ & $1 \cdot 0 \pm 0 \cdot 2$ & CGSC 6629 \\
\hline $\mathrm{N} 43 / \mathrm{pBX} 1$ & $\operatorname{acr} A$ overexpression & $1 \cdot 2 \pm 0 \cdot 4$ & This study \\
\hline SJ261/pEMR2.1 & emr $A, B$ overexpression & $1 \cdot 1 \pm 0 \cdot 2$ & Kim Lewis \\
\hline
\end{tabular}

the activity of drug efflux translocases does not appear to alter the susceptibility of E. coli to PNAs.

\section{Conclusions}

Induction of lacZ by an anti-lacI PNA provides a positive assay for antisense effects. In this study the assay was used to learn which cell-barrier components limit PNA cell entry and antisense effects in E. coli. The results, together with our previous work, provide three lines of evidence indicating that the LPS layer is a major barrier against PNA uptake. First, the LPS mutant strains AS19 and D22 are more susceptible to PNA than wild-type. Second, mutant E. coli strains that are more susceptible to PNA also are more permeable to the antibiotic nitrocefin. Third, chemical agents that compromise LPS integrity can also increase cellular PNA susceptibility. Fortunately, for practical applications, there are opportunities to modify PNAs to better permeate the outer-membrane LPS layer. Also, the activity of multidrug resistance translocases did not significantly alter the susceptibility of E. coli to PNA. This resistance against active efflux, along with evidence for PNA stability in biological fluids, suggests that PNAs that are able to gain access into bacteria are likely to remain active within cells. Therefore, efforts to improve the technology can focus on achieving improved uptake. Finally, the observation that an antisense PNA can upregulate, as well as down-regulate, gene expression supports the idea that antisense PNAs can provide sequence specific tools for the analysis of microbial gene expression and function.

\section{ACKNOWLEDGEMENTS}

This research was supported by the Swedish Foundation for Strategic Research, Pharmacia Corp. and the Danish Biotechnology Programme. We thank Mary Berlyn, Kevin
Bertrand, Ole Micheson, Kim Lewis and Hans Boman for providing E. coli strains and plasmids.

\section{REFERENCES}

Angus, B. L., Carey, A. M., Caron, D. A., Kropinski, A. M. \& Hancock, R. E. (1982). Outer membrane permeability in Pseudomonas aeruginosa: comparison of a wild-type with an antibioticsupersusceptible mutant. Antimicrob Agents Chemother 21, 299-309.

Beja, O. \& Bibi, E. (1996). Functional expression of mouse Mdr1 in an outer membrane permeability mutant of Escherichia coli. Proc Natl Acad Sci U S A 93, 5969-5974.

Blattner, F. R., Plunkett, G., 3rd, Bloch, C. A. \& 14 other authors (1997). The complete genome sequence of Escherichia coli K-12. Science 277, 1453-1474.

Chunhong, L., Peters, A. S., Meredith, E. L., Allman, G. W. \& Savage, P. B. (1998). Design and syntheses of potent sensitizers of gram-negative bacteria based on cholic acid scaffolding. J Am Chem Soc 120, 2961-2962.

Christensen, L., Fitzpatrick, R., Gildea, B., Petersen, K. H., Hansen, H. F., Koch, T., Egholm, M., Buchardt, O. \& Nielsen, P. E. (1995). Solid-phase synthesis of peptide nucleic acids. J Peptide Sci 3, 175-183.

Demidov, V., Potaman, V. N., Frank-Kamenetskii, M. D., Buchardt, O., Egholm, M. \& Nielsen, P. E. (1994). Stability of peptide nucleic acids in human serum and cellular extracts. Biochem Pharmacol 48, 1309-1313.

Ecker, D. J. \& Freier, S. M. (1998). PNA, antisense, and antimicrobials. Nat Biotechnol 6, 332.

Egholm, M., Buchardt, O., Christensen, L. \& 7 other authors (1993). PNA hybridizes to complementary oligonucleotides obeying the Watson-Crick hydrogen bonding rules. Nature $\mathbf{3 6 5}$, 566-568.

Gerberick, G. F. \& Castric, P. A. (1980). In vitro susceptibility of Pseudomonas aeruginosa to carbenicillin, glycine, and ethylenediaminetetraacetic acid combinations. Antimicrob Agents Chemother 17, 732-735. 
Good, L. \& Nielsen, P. E. (1998a). Inhibition of translation and bacterial growth by peptide nucleic acid targeted to ribosomal RNA. Proc Natl Acad Sci U S A 95, 2073-2076.

Good, L. \& Nielsen, P. E. (1998b). Antisense inhibition of gene expression in bacteria by PNA targeted to mRNA. Nat Biotechnol 16, 355-358.

Hancock, R. E. W. (1997). The bacterial outer membrane as a drug barrier. Trends Microbiol 5, 37-42.

Hanvey, J. C., Peffer, N. J., Bisi, J. E. \& 12 other authors (1992). Antisense and antigene properties of peptide nucleic acids. Science 258, 1481-1485.

Helander, I. M., Alakomi, H. L., Latva-Kala, K. \& Koski, P. (1997). Polyethyleneimine is an effective permeabilizer of gram-negative bacteria. Microbiology 143, 3193-3199.

Jensen, K. K., Ørum, H., Nielsen, P. E. \& Nordén, B. (1997). Kinetics for hybridization of peptide nucleic acids (PNA) with DNA and RNA studied with the BIAcore technique. Biochemistry 36, 5072-5077.

Knudsen, H. \& Nielsen, P.E. (1996). Antisense properties of duplex- and triplex-forming PNAs. Nucleic Acids Res 24, 494-500.

Lewis, K. (1994). Multidrug resistance pumps in bacteria: variations on a theme. Trends Biochem Sci 19, 119-123.

Miller, J. H. (1972). Experiments in Molecular Genetics. Cold Spring Harbor, NY: Cold Spring Harbor Laboratory.

Motohashi, N., Kurihara, T., Kawasw, M. \& 7 other authors (1997). Drug resistance reversal, anti-mutagenicity and antiretroviral effect of phthalimido- and chloroethyl-phenothiazines. Anticancer Res 17, 3537-3544.

Ng, E. Y., Trucksis, M. \& Hooper, D. C. Quinolone resistance mediated by nor A: physiologic characterization and relationship to $f l q B$, a quinolone resistance locus on the Staphylococcus aureus chromosome. Antimicrob Agents Chemother 38, 1345-1355.

Nielsen, P. E. \& Haaima, G. (1997). Peptide nucleic acid (PNA). A DNA mimic with a pseudopeptide backbone. Chem Soc Rev 96, 73-78.

Nielsen, P. E., Egholm, M., Berg, R. H. \& Buchardt, O. (1991). Sequence selective recognition of DNA by strand displacement with a thymine-substituted polyamide. Science 254, 1497-1500.

Nikaido, H. (1994). Prevention of drug access to bacterial targets: permeability barriers and active efflux. Science 264, 382-388.
Normark, S., Boman, H. G. \& Matsson, E. (1969). Mutant of Escherichia coli with anomalous cell division and ability to decrease episomally and chromosomally mediated resistance to ampicillin and several other antibiotics. J Bacteriol 97, 1334-1342.

Norton, J. C., Piatyczek, J. A., Wright, W. E., Shay, J. W. \& Corey, D. R. (1996). Inhibition of human telomerase activity by peptide nucleic acid. Nat Biotechnol 14, 615-619.

Püschl, A., Sforza, S., Haaima, G., Dahl, O. \& Nielsen, P. E. (1998). Peptide nucleic acids (PNAs) with a functional backbone. Tetrahedron Lett 39, 4707-4710.

Sekiguchi, M. \& lida, S. (1967). Mutants of Escherichia coli permeable to actinomycin. Proc Natl Acad Sci USA 58, 2315-2320.

Shafer, W. M., Qu, X., Waring, A. J. \& Lehrer, R. I. (1998). Modulation of Neisseria gonorrhoeae susceptibility to vertebrate antibacterial peptides due to a member of the resistance/ nodulation/division efflux pump family. Proc Natl Acad Sci U S A 95, 1829-1833.

Simons, R. W. \& Kleckner, N. (1988). Biological regulation by antisense RNA in prokaryotes. Annu Rev Genet 22, 567-600.

Sieradzki, K. \& Tomasz, A. (1997). Suppression of beta-lactam antibiotic resistance in a methicillin-resistant Staphylococcus aureus through synergic action of early cell wall inhibitors and some other antibiotics. J Antimicrob Chemother 39, suppl. A, 47-51.

Taylor, R. W., Chinnery, P. F., Turnbull, D. M. \& Lightowlers, R. N. (1997). Selective inhibition of mutant human mitochondrial DNA replication in vitro by peptide nucleic acids. Nat Genet 15, 212-215.

Vaara, M. \& Jaakkola, J. (1989). Sodium hexametaphosphate sensitizes Pseudomonas aeruginosa, several other species of Pseudomonas, and Escherichia coli to hydrophobic drugs. Antimicrob Agents Chemother 33, 1741-1747.

Wittung, P., Nielsen, P. E., Buchard, O., Egholm, M. \& Nordén, B. (1994). DNA-like double helix formed by peptide nucleic acid. Nature 368, 561-563.

Zorzopulos, J., de Long, S., Chapman, V. \& Kozloff, L. M. (1989). Evidence for a net-like organization of lipopolysaccharide particles in the Escherichia coli outer membrane. FEMS Microbiol Lett 52, 23-26.

Received 2 May 2000; revised 21 June 2000; accepted 4 July 2000. 